\title{
A Traffic Flow Approach to Early Detection of Gathering Events
}

\author{
Xun Zhou ${ }^{1}$, Amin Vahedian Khezerlou ${ }^{1}$, Alex Liu' ${ }^{2}$, Zubair Shafiq ${ }^{1}$, Fan Zhang ${ }^{3}$ \\ ${ }^{1}$ The University of lowa, lowa City, IA 52242 \\ \{xun-zhou,amin-vahediankhezerlou,zubair-shafiq\}@uiowa.edu \\ ${ }^{2}$ Michigan State University, East Lansing, MI 48824 \\ alexliu@cse.msu.edu \\ ${ }^{3}$ SIAT, Chinese Academy of Sciences, Shenzhen, China \\ zhangfan@siat.ac.cn
}

\begin{abstract}
Given a spatial field and the traffic flow between neighboring locations, the early detection of gathering events (EDGE) problem aims to discover and localize a set of most likely gathering events. It is important for city planners to identify emerging gathering events which might cause public safety or sustainability concerns. However, it is challenging to solve the EDGE problem due to numerous candidate gathering footprints in a spatial field and the non-trivial task to balance pattern quality and computational efficiency. Prior solutions to model the EDGE problem lack the ability to describe the dynamic flow of traffic and the potential gathering destinations because they rely on static or undirected footprints. In contrast, in this paper, we model the footprint of a gathering event as a Gathering directed acyclic Graph (G-Graph), where the root of the G-Graph is the potential destination and the directed edges represent the most likely paths traffic takes to move towards the destination. We also proposed an efficient algorithm called SmartEdge to discover the most likely non-overlapping G-Graphs in the given spatial field. Our analysis shows that the proposed G-Graph model and the SmartEdge algorithm have the ability to efficiently and effectively capture important gathering events from real-world human mobility data. Our experimental evaluations show that SmartEdge saves $50 \%$ computation time over the baseline algorithm.
\end{abstract}

\section{CCS Concepts}

-Information systems $\rightarrow$ Geographic information systems;

\section{Keywords}

Gathering Event; Early Detection; Spatial Data Mining

\section{INTRODUCTION}

Background \& Motivation. A gathering event is the process where a large number of moving objects (e.g., taxi

Permission to make digital or hard copies of all or part of this work for personal or classroom use is granted without fee provided that copies are not made or distributed for profit or commercial advantage and that copies bear this notice and the full citation on the first page. Copyrights for components of this work owned by others than ACM must be honored. Abstracting with credit is permitted. To copy otherwise, or republish, to post on servers or to redistribute to lists, requires prior specific permission and/or a fee. Request permissions from permissions@acm.org.

SIGSPATIAL'16, October 31-November 03, 2016, Burlingame, CA, USA

(C) 2016 ACM. ISBN 978-1-4503-4589-7/16/10.. \$15.00

DOI: http://dx.doi.org/10.1145/2996913.2996998 cabs, pedestrians) arrive at a specific destination during a time period via different paths. Typical examples of gathering events include but are not limited to: (1) a traffic congestion where more-than-usual number of vehicles arrive at a specific road segment or intersection and are not able to leave during the same time period, (2) fans arriving at the vicinity of a stadium before the game starts, and (3) protesters gather at a destination (e.g., a park) in a planned or unplanned social protest. Gathering events can have a significant impact on urban planning and public safety. For example, traffic congestion leads to extra gas emissions and low transportation efficiency. As another example, social activities such as sports events may put a strain on public resources (e.g., parking spaces, cellular capacity) and may even become a threat to public safety. Although many gathering events are predictable and occur regularly (e.g., football games, concerts), some others are rare or unexpected. Even for planned gathering events, the traffic volume may far exceed the expectation. For example, the stampede in Shanghai in 2014 was a result of larger-than-expected crowd gathering for the New Year's Eve [16]. Therefore, it is important for city planners and other stakeholders to have the ability to identify gathering events as early as possible.

Problem Statement. This paper investigates a computational solution to detecting gathering events based on human mobility data. Specifically, this paper is focused on detecting the footprint of gathering events where the total amount of moving objects is much higher than expected. Given a spatial framework that partitions the space into grids and expected and observed traffic flow between neighboring grids, the Early Detection of Gathering Event (EDGE) problem aims to discover top- $k$ most likely gathering events, their destinations, and the most likely routes along which moving objects gather at the destination. The EDGE problem is challenging to solve because the total number of possible event footprints in a spatial framework grow exponentially as a function of the number of spatial grids in the study area, and it is non-trivial to balance the quality of results (e.g., statistical significance) and computational efficiency.

Limitations of Prior Art. Prior work on event detection can be broadly categorized into two groups. The first group of methods are limited to identifying events with regularlyshaped footprints $[5,4,10,12]$ (e.g., circular, rectangular). The second group of methods are limited to finding undirected graph footprints (e.g., black holes or volcanoes [3, 9, 
8]). The key limitation of the prior work is that they lack the ability to capture how moving objects gather towards a specific destination. Therefore, prior work cannot precisely characterize gathering events.

Proposed Approach. To address the limitations of prior work, we model the footprint of a gathering event as a Gathering directed acyclic Graph (G-Graph) to better characterize the path taken by the traffic in the gathering event. The root of the G-Graph (grid with only incoming edges) represents the location of a potential gathering event and the branches are the most likely paths used to reach the destination. We propose a novel metric called Gathering Score (GScore) to quantify the likelihood that more-than-expected traffic is moving towards the root of a G-Graph while the traffic moving away from the root is not more than expected. We also propose SmartEdge algorithm to efficiently discover a set of $k$ non-overlapping G-Graphs with the highest GScore in the underlying spatial field.

Technical Challenges. It is computationally challenging to discover the top- $k$ non-overlapping G-Graphs due to the following reasons. First of all, the number of possible paths from a grid to the root of a G-Graph increases exponentially with respect to path length. Exhaustively enumerating all the paths to generate a G-Graph is computationally prohibitive. Second, it is infeasible to build a G-Graph for every grid due to the high cost of building a G-Graph and the large number of grids. Third, G-Graphs may overlap with each other and produce redundant results. Correctly and efficiently reducing the results to the $k$ most likely nonoverlapping G-Graphs is non-trivial.

Key Contributions. In this paper we make the following contributions: (1) We formulate the problem of detecting gathering events as a computational problem. We propose to use a novel concept called G-Graph to model the footprint of gathering events. We also propose a measure GScore to effectively quantify the likelihood that traffic is moving towards the root of a G-Graph. (2) We proposed SmartEdge algorithm to efficiently discover top- $k$ non-overlapping G-Graphs. (3) We provide a case study on real taxi data from Shenzhen, China. The proposed algorithm captured important gathering events such as a pop concert in the city stadium. We also conducted experimental evaluations using a month's taxi trajectory data. SmartEdge outperforms the baseline algorithm in computation time by more than $50 \%$.

The paper is organized as follows. Section 2 presents the concepts and definitions, followed by a problem statement. Computational Solutions to the EDGE problem are discussed in Section 3. Section 4 presents a case study using taxi trajectory data. Experimental evaluation on computational efficiency are presented in Section 5. Related work are discussed in Section 6. Finally Section 7 concludes the paper.

\section{PROBLEM FORMULATION}

\subsection{Overview of Gathering Events Detection}

The EDGE problem formulation is based on the following traffic monitoring workflow: the real-time traffic flow of the entire region is monitored. High-volume traffic flows crossing the boundaries of adjacent regions are identified. Then an algorithm finds the most likely destination of the traffic (if any) as well as a flow map of the traffic to the destination. This graph shows the most likely gathering footprints.

\subsection{Concepts and Definitions}

A spatial field $S$ is a two-dimensional region (e.g., a city) partitioned into grid cells $s_{1}, s_{2}, \ldots s_{n}$. Given a spatial field, the location of a moving object (e.g., taxi) at a certain time thus could be mapped to one of the grids.

A directed edge $e=\left(s_{i}, s_{j}\right)$ can be defined between each pair of adjacent grids $s_{i}$ and $s_{j}$. Given a certain time interval (e.g., 19:40-19:50, August 1, 2013), the observed traffic flow along $e$ (denoted as $C_{e}$ ) is a non-negative integer that measures the number of moving objects (e.g., taxis) going from $s_{i}$ to $s_{j}$. In reality, traffic flows can be obtained from sensing devices such as loop detectors or by processing realtime GPS locations of moving objects.

Next we propose a mechanism to quantify the abnormality of traffic flow observed on each edge. Poisson distribution is commonly used to model the number of discrete events such as the total number of car arriving at an intersection during a time interval. For each edge, we use a Chi-square test to fit a Poisson distribution on its historical traffic flow data. Results show that the observed traffic flows along an edge in the same time interval of day can be well approximated with a Poisson distribution with mean equals the average of the observed traffic. We call this mean the baseline traffic flow of edge $e$ (denoted as $B_{e}$ ).

Various tests have been developed to identify statistically significant anomalous patterns. For example, Kulldroff's Spatial Scan Statistics [7] is a classic model for data with Poisson distributions, where the likelihood ratio between alternative hypothesis $H_{1}$ (risk inside a region is higher than outside) and the null hypothesis $H_{0}$ (risk is the same everywhere) is maximized over all the possible regions. Here we employ the idea used by Neill [10], which simplified Kulldroff's spatial scan statistic model. Neill et al proposed an Expectation-based Poisson (EBP) model, which compares the observed value of a region with its own historical average instead of the counts outside. We employ the idea of $\mathrm{EBP}$ in our problem and propose the following hypothesis testing mechanism.

Assume $C_{e}$ is the observed traffic flow along $e$ in a time interval $t$, and $B_{e}$ is the baseline traffic flow in the same time interval of day. Under the null hypothesis $H_{0}$, the observed flow $C_{e}$ is drawn from $\operatorname{Poisson}\left(B_{e}\right)$. The alternative hypothesis $H_{1}$ is: the observed flow $C_{e}$ is drawn from a different Poisson distribution with an elevated mean value $q B_{e}$ where $q \geq 1$. The EBP test maximizes the likelihood ratio between $H_{1}$ and $H_{0}\left(\frac{\operatorname{Pr}\left(C_{e} \mid H_{1}\right)}{\operatorname{Pr}\left(C_{e} \mid H_{0}\right)}\right)$ when $q=\frac{C_{e}}{B_{e}}\left(C_{e} \geq\right.$ $B_{e}$ ). When $C_{e}<B_{e}$ (i.e., the observed flow is lower than the expected), the score is set to 0 . Note when $B_{e}=0$ (e.g., no road or inside a park), the corresponding edge is removed from the spatial field thus not included in any calculation. The log likelihood ratio of the given observed flow thus can be expressed as follows:

$$
\operatorname{LLR}(e)= \begin{cases}C_{e} \log \frac{C_{e}}{B_{e}}+\left(B_{e}-C_{e}\right) & \text { if } C_{e} \geq B_{e} \\ 0 & \text { otherwise }\end{cases}
$$

Definition 1. A Significant Flow in spatial field $S$ in a time interval $t$ is an edge $e=\left(s_{i}, s_{j}\right)$ such that $\operatorname{LLR}(e)>0$ and $\operatorname{LLR}(\mathrm{e})$ is statistically significant at $\alpha$ level.

The statistical significance of a LLR score is typically assessed via Monte-Carlo simulations to filter results generated by random chance. Each trial of the simulation will 
generate a random observation $C_{\text {rand }}$ under null hypothesis $H_{0}$ and calculates the corresponding score $L L R_{\text {rand }}(e)$. The actual score $\operatorname{LLR}(e)$ is significant at $\alpha$ level if no more than $\alpha * 100 \%$ of all the simulated score $\operatorname{LLR}_{\text {rand }}(e)$ are as high as $\operatorname{LLR}(e)$. However, Monte-Carlo simulation is very computationally expensive. Due to the properties of the log likelihood ratio score, a closed form of the p-value can be derived thus avoiding Monte-Carlo simulations.

Lemma 1. Given two edges $e_{1}$ and $e_{2}$ with the same baseline flow $B$ and different observed flows $C_{e_{1}}$ and $C_{e_{2}}\left(C_{e_{1}} \geq\right.$ $B$ and $\left.C_{e_{2}} \geq B\right)$, respectively, $\operatorname{LLR}\left(e_{1}\right) \geq \operatorname{LLR}\left(e_{2}\right) \Longleftrightarrow$ $C_{e_{1}} \geq C_{e_{2}}$.

Proof. The partial derivative $\frac{d L L R(e)}{d C_{e}}=\log \frac{C_{e}}{B_{e}}>0$ shows that the $\operatorname{LLR}(\mathrm{e})$ function is monotonically increasing with increasing $C_{e}$ and fixed $B_{e}$ when $C_{e} \geq B_{e}$. Thus the lemma is proved.

$\operatorname{LLR}(e)$ is significant at $\alpha$ level means: the chance that the score $L L R_{\text {rand }}(e)$ calculated based on randomly generated observation $C_{\text {rand }}$ under $H_{0}$ is no less than the real $\operatorname{LLR}(e)$ is at most $\alpha$. According to Lemma $1, L L R_{\text {rand }}(e) \geq L L R(e)$ when and only when the $C_{\text {rand }} \geq C_{e}$. In other words, for a LLR(e) to be significant at 0.005 level, the actual $C_{e}$ must be no lower than at least $99.5 \%$ of the random $C_{\text {rand }}$ drawn from $\operatorname{Poisson}\left(B_{e}\right)$. This can be quickly tested in constant time by comparing $1-\operatorname{Pr}\left(X<C_{e}\right)$ with $\alpha$. LLR(e) being tested is significant at $\alpha$ level when $1-\operatorname{Pr}\left(X<C_{e}\right) \leq \alpha$.

Given a significant flow $e$ and a potential destination $r$, we evaluate how likely it is to find a path connecting $e$ and $r$ such that the traffic along this path is higher than normal.

Definition 2. Given a grid $r$ and a significant flow $e_{i n}=$ $\left(s_{i}, s_{j}\right)$, an incoming path $p_{i n}$ of $r$ from $e_{i n}$ is a sequence of directed edges $e_{i n}, e_{1}, e_{2}, \ldots, e_{n}$ such that $p_{i n}$ is a shortest path in Manhattan distance from $s_{j}$ to $r$. $e_{i n}$ is called an incoming significant flow of $r$. $\operatorname{dist}\left(r, e_{i n}\right)=\operatorname{dist}\left(r, s_{j}\right)$.

The shortest path constraint is added based on the assumption that most of the moving objects and vehicles should take the shortest path when gathering towards a destination. Note sometimes there might not exist a shortest path between a significant flow and a destination. For example in Figure 1(a) there is no incoming path from $e_{2}$ to $r$. In such cases we may define an outgoing path along which traffic goes out of $r$ to $e_{s i g}$.

Definition 3. Given a grid $r$ and a significant flow $e_{\text {out }}=$ $\left(s_{i}, s_{j}\right)$, an outgoing path $p_{\text {out }}$ of $r$ to $e_{\text {out }}$ is a sequence of directed edges $e_{1}, e_{2}, \ldots, e_{\text {out }}$ such that $p_{\text {out }}$ is a shortest path in Manhattan distance from $r$ to $s_{i}$. $e_{\text {out }}$ is called an outgoing significant flow of $r$. $\operatorname{dist}\left(r, e_{\text {out }}\right)=\operatorname{dist}\left(r, s_{i}\right)$.

Note that a significant flow cannot be both incoming and outgoing for the same grid $r$ since the directed shortest path between them is unique. Figure 1(a) shows an example of incoming paths and outgoing paths. $e_{1}$ and $e_{2}$ are two significant flows. There exist two possible incoming path from $e_{1}$ to $r$ (solid, pink arrows) and two possible outgoing paths from $r$ to $e_{2}$ (dashed, green arrows).

Next we quantify the likelihood that a path has a high traffic. One possible way might be to employ the idea used in the Spatial Scan Statistic and EBP. They assumed that all the locations (edges in our case) inside the event footprint (a path in our case) have a uniform elevation $q$. Then the sum of baseline $\sum B_{e}$ and the sum of observed counts $\sum C_{e}$
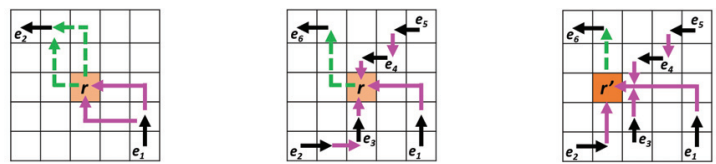

(a) Incoming and (b) A G-Graph (c) A G-Graph outgoing paths

rooted at $r$

rooted at $r^{\prime}$

\section{Figure 1: Examples of Paths and G-Graphs}

are used to calculate the LLR score of a path using Equation 1. This idea, however, does not work well in our case. Even in the same gathering event, different edges may have different degrees of traffic increase due to merging and splitting of traffic. Assuming same elevation may significantly over-estimate the likelihood. Alternatively, we choose the below score definition, which maximizes the log-likelihood ratio for each edge separately to allow them to have different traffic elevation $q$. The score tests how likely every single flow along a path $p$ is higher than their respective baseline values. Naturally, we could multiply the likelihood ratio score of each flow along $p$, which is equivalent to the sum of the log-likelihood ratio of every flow along $p$. Formally, it can be expressed as follows:

$$
L L R(P)=\sum_{e \in P} \operatorname{LLR}(e)
$$

Definition 4. The most likely incoming (outgoing) path $P_{\text {in }}^{*}(e, r)\left(P_{\text {out }}^{*}(e, r)\right)$ between $r$ and $e$ is the incoming (outgoing) path with the maximum LLR score.

A grid location is likely to be the destination of a gathering event when the likelihood of every edge along its incoming paths having an elevated traffic volume is high, while the likelihood of every edge along outgoing paths having an elevated traffic volume is low. This is to ensure that we do not find false alarms such as an intersection where both incoming and outgoing paths have elevated traffic amount. Note that not all the significant flows in the study area should be linked with every potential destination. High traffic volume in a region is very unlikely to be part of a gathering event occurring 20 kilometers away in an urban environment. Here we define a maximum distance $d$, where only significant flows within distance $d$ to the grid should be included.

The gathering score (GScore) of a grid is thus calculated by summing the log likelihood ratio of each distinct flow along incoming paths with length $d$ or shorter, and subtracting the log likelihood ratio of each distinct flow along the outgoing paths with length $d$ or shorter. Formally, the GScore is defined as follows:

$$
G S c o r e(r)=\sum_{e \in P_{\text {in }}^{*}\left(e_{\text {in }}, r\right)} \operatorname{LLR}(e)-\sum_{e \in P_{\text {out }}^{*}\left(e_{\text {out }}, r\right)} \operatorname{LLR}(e)
$$

Definition 5. Gathering Graph (G-Graph). Given a root grid $r \in S$, and all the significant flows $E_{\text {sig }}$ such that $\operatorname{dist}\left(e_{s i g}, r\right) \leq d, e_{s i g} \in E_{\text {sig }}$ at time interval $t$, a Gathering Graph (G-Graph) rooted at $r$ is a directed acyclic graph $G(r)$ whose vertices are the grids and edges are the flows, and $G=\bigcup_{e \in E \text { sig }} P_{i n}^{*}(r, e)$. G.GScore $=$ GScore $(r)$.

Given the above definitions, one may identify very similar G-Graphs rooted at grids close to each other which overlap heavily. Figure 1(b) and Figure 1(c) show two different GGraphs with the same set of significant flows. Note the outgoing paths (green) are not part of the G-Graphs. These 
G-Graphs usually represent the same gathering event and may not provide much additional useful information. Also if two G-Graphs share a flow, it is hard to tell which root is the actual destination of this flow. To this end, we only find G-Graphs with the highest score among all the G-Graphs within $2 d$ distance so that there is absolutely no overlapping among the G-Graphs.

Definition 6. Given two G-Graphs $G_{1}(r)$ and $G_{2}\left(r^{\prime}\right)$ with depth $d, G_{1}$ dominates $G_{2}$ if $G_{1}$.GScore $>G_{2}$.GScore and $\operatorname{dist}\left(r, r^{\prime}\right) \leq 2 d$.

Finally, we would like to find a set of G-Graphs that are not dominated by others and have the highest scores among all the candidates. Hereby we define the dominant G-Graph set as follows:

Definition 7. A $k$-dominant G-Graph set $\hat{G}_{k}$ is a set of no more than $k$ G-Graphs such that none of them dominate each other, and for any G-Graph $G^{\prime} \notin \hat{G}_{k}$, one of the following conditions hold: (1) $G^{\prime}$.GScore $<\operatorname{Min}_{G \in \hat{G}_{k}}\{$ G.GScore $\}$, or (2) $\exists G \in \hat{G}_{k}$ such that $G$ dominates $G^{\prime}$.

\subsection{Problem Statement}

Given the above definitions, the EDGE problem could be formulated as follows:

Given:

- A spatial field $S$ with observed and baseline flows $C, B$ during time interval $t$

- Maximum distance threshold $d$

- $p$-value threshold $\alpha$

- Size of result $k$

Find:

- The $k$-dominant G-Graph set for time interval $t$

Objective:

- Reduce Computational Cost

Constraints:

- All the distances are measured in Manhattan distance

- Correctness and Completeness

\section{COMPUTATIONAL SOLUTION 3.1 A Brute-Force Algorithm}

The EDGE problem can be solved using straightforward ideas following the workflow discussed in Section 2.1. Algorithm 1 presents the pseudo code of a brute-force algorithm. First, each edge is examined and significant flows are identified (Line 1). Then the algorithm constructs the G-Graph rooted at each grid. For each candidate root grid $r$, all the significant flows within distance $d$ are fetched. Then an exhaustive search is performed to find the most likely paths to/from $r$. The corresponding G-score is also calculated (Line 2-14). Finally the G-Graphs generated are sorted and scanned. Only the top $k$ G-Graphs whose are not $d$-dominated by any others are reported (Line 15-20).

\subsection{The Smart Edge Algorithm}

There are several computation bottlenecks in the BruteForce algorithm. (1) A grid may be the root of a G-Graph only when there is at least one significant flow within distance $d$. Many candidate roots won't even qualify to have a G-Graph.(2) It is costly to use exhaustive search to find the most likely path for each significant flow. (3) The algorithm does not have any ability to prune candidate GGraphs. Since we are only interested in the top- $k$ dominant G-Graphs, candidates with very low GScores or dominated by others should not be generated.

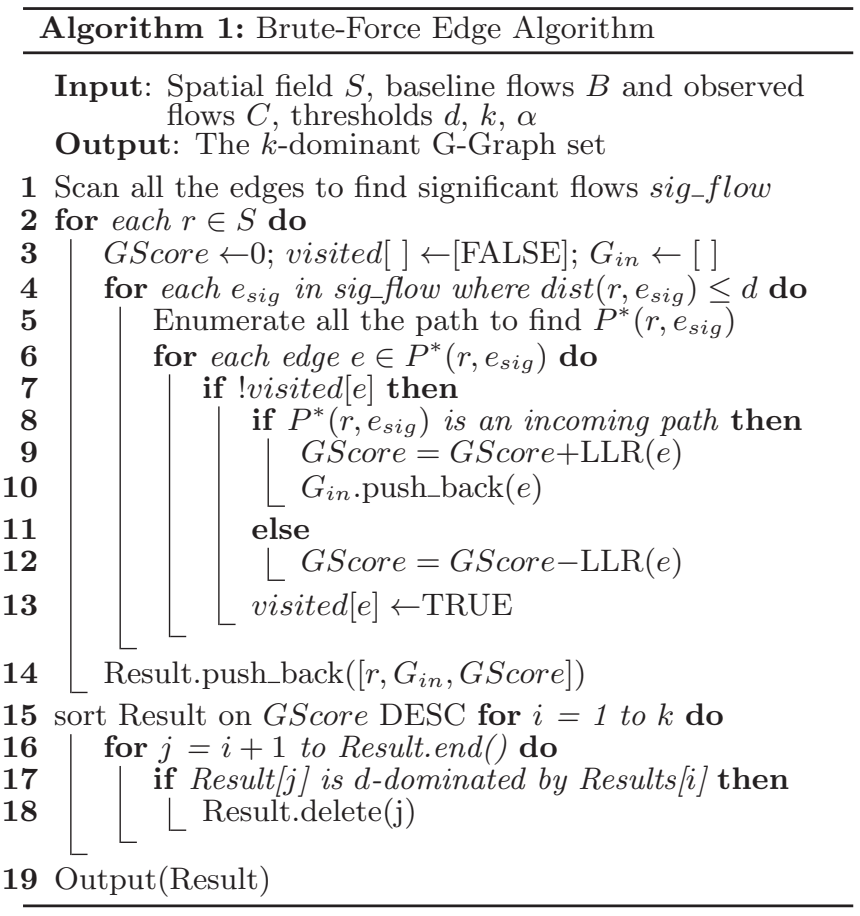

To address the above computational bottlenecks, we present a new algorithm SmartEdge with three design decisions for better computational efficiency.

\subsubsection{Candidate Root Filter}

As previously mentioned, locations with no significant flow within distance $d$ cannot be the root of a G-Graph. A location $r$ is a possible root of the G-Graph that includes significant flow $e_{\text {sig }}$ only if $\operatorname{dist}\left(r, e_{s i g}\right) \leq d$. Figure $2(\mathrm{a})$ shows an example where the yellow area are the possible root locations with an incoming path from significant flow $e$, and the blue area are the possible root locations with an outgoing path to $e$ (assume $d=4$ ).

We create a data structure called the Candidate Root Index (CRI) with a hash table to store candidate roots with at least one significant flow around. Each root element is also linked with two vectors that store all the incoming and outgoing significant flows within distance $d$ to the root. The time to locate significant flows when generating G-Graphs is reduced to constant.

Since we will need the number of significant flows at each distance to the root in later calculation of the score upper bound, we store the significant flows separately in $d+1$ bins based on their distance to the root. The total number of significant flows in each bin and the total number of significant flows near the root are also recorded. When a significant flow $e_{\text {sig }}$ is identified, the algorithm finds all the locations that could be the root of $e_{\text {sig }}$. For each of these candidates, the algorithm calculates its distance from $e_{s i g}$ and inserts $e_{s i g}$ into the corresponding bin. The count for that bin as well as the total count are incremented by 1 . Locations with no significant flow around will not exist in the CRI thus won't be evaluated. Figure 2(b) shows the structure of the CRI.

\subsubsection{Building G-Graph with Dynamic Programming}

Due to the way the LLR score of a path is defined, the most likely path between a candidate root grid $r$ and a sig- 


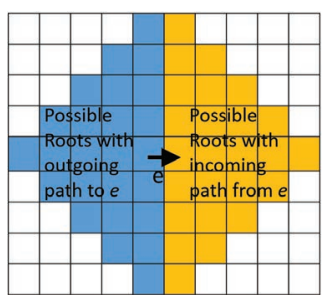

(a) Possible root locations of a significant flow $(d=4)$

Figure 2: Candidate Root Filter Examples (best viewed in color).

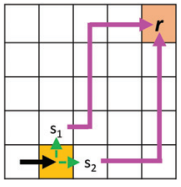

(a) An example of DP GGraph generation optimal sub-structure

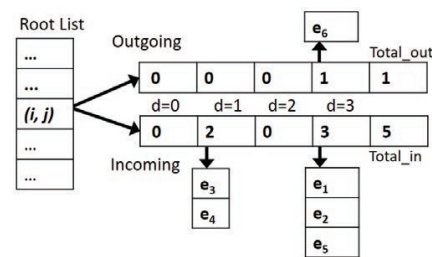

(b) Candidate Root Index
Figure 3: G-Graph Generation using Dynamic Programming.

nificant flow $e=\left(s_{a}, s_{b}\right)$ could be calculated using a dynamic programming approach. The following optimal substructure can be observed. Assuming $r . x>s_{b} . x, r . y>s_{b} . y$, the most likely path $P^{*}$ from grid $s_{b}$ to root $r=(r . x, r . y)$ can be calculated by comparing the best paths to $r$ via $(r . x-1, r . y)$ and $(r . x, r . y-1)$. This optimal substructure can be recursively used for $(r . x-1, r . y)$ and $(r . x, r . y-1) \ldots$ until the best path is found. Figure 3(a) shows an example where the best path connecting the significant edge (black arrow) and the root (red grid) is chosen between the two pink routes, either via grid $s_{1}$ or via $s_{2}$. Best paths from $s_{1}$ to $r$ and from $s_{2}$ to $r$ can be calculated in the same way recursively.

When building a G-Graph, we use an array $P$ to keep (1) the maximum total LLR score from each grid to the root, and (2) the next grid along the corresponding most likely path. Algorithm 2 presents the pseudo code of the new procedure Build_GGrap_DP. For a given root $r$ and a list of significant flows nearby (fetched from the Candidate Root Index), the algorithm picks each significant flow and traverse all the grids in the rectangular area bounded by the root and this significant flow in a breadth-first manner, starting from the root (Line 2-7). The most likely path to the root from every grid is calculated until the significant flow is reached. After finding the most likely path, all the flows along this path will be added to the G-Graph (Line 8-14). Figure 3(b) shows the breadth-first traversal order to calculate the max total LLR score and the best path to/from $r$ at each location.

\subsubsection{G-Graph Pruning: GScore Upper-Bound}

Finally we discuss how to efficiently prune candidate GGraphs without generating them to save computation time. The G-Graph generation step takes up to $O\left(\left|E_{\text {sig }}\right| d^{2}\right)$ for each root even with the dynamic programming design decision, where $\left|E_{\text {sig }}\right|$ is the number of significant flows near the current root. In fact, most G-Graphs can be pruned because they are either dominated by a nearby G-Graph or has a lower score than the top- $k$ ones. To address this issue, we propose two ideas (1) a GScore upper-bound for candidate roots, which is easy to compute, and (2) a pruning strategy

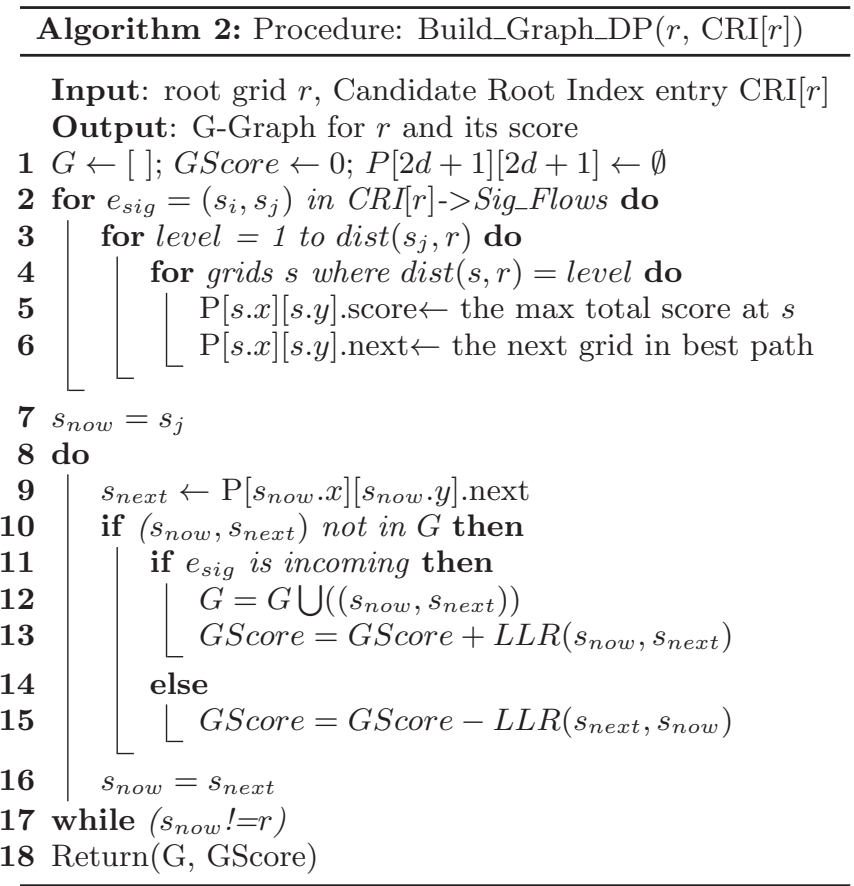

for candidate G-Graphs.

The upper-bound of the GScore of $G(r)$ can be calculated as follows:

$$
\left.\widehat{\operatorname{Score}}(r)=N_{e}(r) \times \widehat{L L R\left(e_{\text {ins }}\right.}\right)+\sum_{e_{\text {sig }} \in E_{\text {sig }}} \operatorname{LLR}\left(e_{\text {sig }}\right)
$$

$N_{e}(r)$ is the upper-bound of the number of insignificant

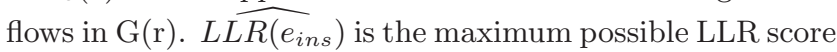
of these insignificant flows. Note we only consider incoming paths when calculating the upper-bounds since the LLR of outgoing paths could be as low as 0 and thus calculating their lower bound won't contribute much to upper-bounds. However, our upper-bounds can still be very effective.

Calculating $L \widehat{L R\left(e_{i n s}\right)}$ : We design a zone-index to keep track of the maximum $\operatorname{LLR}(e)$ of insignificant flows. The entire spatial field $|S|$ is partitioned into zones of size $(2 d+$ $1) \times(2 d+1)$. This size allows a G-Graph to fit in a single zone in the best case, while covers at most 4 zones in the worst case. Each zone keeps the maximum LLR of insignificant flows in each of the four directions. Figure 4 shows an example of the zone-index with $d=2$. There are four zones and the maximum LLR of insignificant flows in each zone are listed on the right. For example, the maximum LLR of insignificant flows pointing to right in zone 1 is 4.00 . These numbers are obtained at the beginning of the algorithm when the LLR score of each directed edge is calculated. Each insignificant flow is mapped to a corresponding zone. If the insignificant flow's LLR score is higher than the current maximum in the zone-index, then the current maximum LLR of the same direction is updated.

When it comes to calculating $L \widehat{L R\left(e_{i n s}\right)}$ for a candidate root $r$, we pull all the incoming significant flows near $r$ from the CRI. For each significant flow $e_{s i g}$, we check the directions and zones of insignificant flows needed to connect $e_{s i g}$ to $r$. Then the maximum LLR of these zones and directions are fetched. For example, $e_{1}$ of $G(r 1)$ can be connected to 


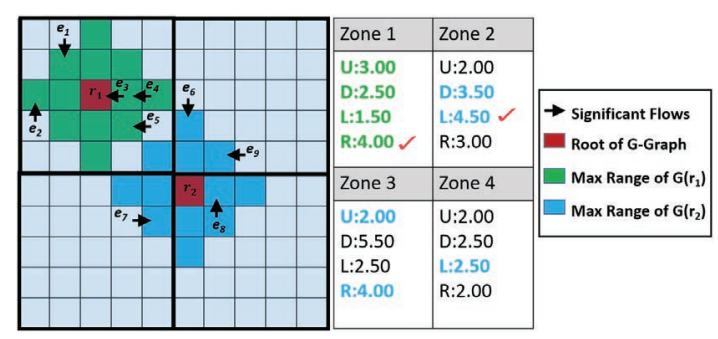

Figure 4: $L \widehat{L R\left(e_{i n s}\right)}$ calculation with zone-index $(d=2)$

$r 1$ via insignificant flows pointing right or down in zone 1 . The corresponding max LLR records are fetched and compared. The max LLR along $P_{i n}^{*}\left(e_{1}, r_{1}\right)$ is thus $\max \{2.50$, $4.00\}=4.00$. The same is done for all the significant flows near $r_{1}$ and $\widehat{L L R\left(e_{i n s}\right)}$ is the max of all the records pulled from the zone-index. It is possible that records in multiple zones are pulled for the same candidate root. In Figure 4, records in green and blue show the records fetched to calculate $L \widehat{L R\left(e_{i n s}\right)}$ of $r_{1}$ and $r_{2}$, respectively. The final results are 4.00 and 4.50 as they are the maximum record fetched for $r_{1}$ and $r_{2}$.

Calculating $N_{e}$ : For every significant flow $e_{s i g}=\left(s_{i}, s_{j}\right)$ within distance $d$ to root $r$, the number of flows needed to connect it to $r$ is $\operatorname{dist}\left(s_{j}, r\right)$. Hence, a loose upper bound for $N_{e}$ is the sum of the distance between each significant flow and $r$, i.e., $N_{e} \leq \sum_{e_{s i g} \in E_{s i g}} \operatorname{dist}\left(s_{j}, r\right)$. However, this upper bound can be tightened since the most likely paths between the significant flows and the root may overlap thus reducing the possible number of distinct flows in G-Graph.

Let $N_{e}(i)$ be the maximum number of distinct flows of any G-Graph at distance $i$ from the root $r(0 \leq i \leq d)$. Due to the optimal substructure discussed previously, the most likely path from each grid to the root $r$ is unique. For a grid location, there is only one most likely path to the root. Thus the total number of distinct flows at distance $i$ to $r$ equals the number of grids at distance $i+1$ since these grids uniquely identify the flows. Hence, $N_{e}(i)=4 d+4$. Figure 5(a) shows a G-Graph with the maximum possible number of edges $(\mathrm{d}=3)$. There are 4,8 , and 12 possible edges within distance 0,1 , and 2 from $r$.

If the total number of significant flows that are $i$ or further from $r$ is more than $N_{e}(i)$, then only $N_{e}(i)$ flows can exist in the final G-Graph. Otherwise, the maximum number of flows at distance $i$ equals the number of significant flows at least $i$ away from $r$ (denoted as $N_{\text {sig }}(i)$ ). Formally, $N_{e}=$ $\sum_{i=0}^{d} \operatorname{Min}\left\{N_{e}(i), N_{\text {sig }}(i)\right\}$. Each $N_{\text {sig }}(i)$ can be calculated by a linear scan of the bin sizes in the Candidate Root Index entry of root $r$ in descending order at cost $O(d)$.

\subsubsection{Candidate G-Graph Pruning Strategy}

Based on the GScore upper-bound discussed above, we show how to prune G-Graphs as early as possible. In general, a candidate root is likely to have higher GScore if there are more incoming significant flows near it. Thus we sort the candidate root index discussed in Section 3.2.1 based on the total number of incoming significant flows in descending order to increase the chance of visiting the dominating GGraphs earlier.

For each candidate root $r$ in the candidate root index

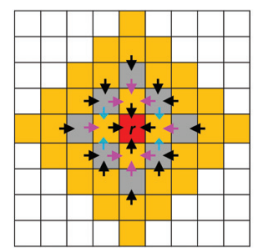

(a) An example of $N_{e}$ with (b) An example of recur$\mathrm{d}=3$

Figure 5: Upper-Bound and G-Graph Pruning Examples

(CRI), a procedure $G_{-}$Prune is called to decide if $r$ should be added to the top- $k$ list or pruned. Algorithm 3 presents its pseudo code. The procedure has access to the CRI and a priority queue $Q$, which keeps the current top- $k$ G-Graphs. The upper-bound $G \widehat{S c o r e}(r)$, if not calculated yet, will be calculated and compared with the minimum GScore in $Q$ (Line 3). If the upper-bound is lower, then $r$ will be pruned (Line 4-6). Otherwise the actual G-Graph rooted at $r$ will be generated using the Build_Graph_DP procedure and the actual GScore(r) will be compared with the minimum GScore in $Q$ again. If GScore(r) is lower then $r$ is pruned (Line 7-11). Otherwise, we may consider adding $G(r)$ into $Q$ and pop the current $k$-th G-Graph.

Before adding $G(r)$ to $Q$, there are some additional issues to consider. If one of $r$ 's neighbors (i.e., within distance $2 d$ ) $r^{\prime}$ has a higher GScore, $G\left(r^{\prime}\right)$ will be added to $Q$ and $G(r)$ will need to be removed from $Q$. Same for $r^{\prime}$. If there is another G-Graph $G\left(r^{\prime \prime}\right)$ such that $G\left(r^{\prime \prime}\right)$ dominates $G\left(r^{\prime}\right)$ but does not dominate $G(r)$ then $G\left(r^{\prime \prime}\right)$ will be added to $Q$ and $G\left(r^{\prime}\right)$ will need to be removed. Also $G(r)$ should be added back to $Q$. This issue may exist for every candidate G-Graph. In the worst case there may exists a long chain of G-Graphs with such dominating relationship from one end to the other. Adding and removing G-Graphs to and from $Q$ takes huge computation time. To resolve this issue, we do not push $G(r)$ above into $Q$ until we can verify that $G(r)$ is not dominated by any other G-Graphs that are either already in $Q$ or will be pushed into $Q$. Specifically, we examine every candidate root $r^{\prime}$ within $2 d$ distance to $r$ and get their GScore upper-bounds. If the upper-bound of GScore $\left(r^{\prime}\right)$ is higher than GScore $(r)$ then $G\left(r^{\prime}\right)$ is built and $r^{\prime}$ is pushed into a list $M$ (Line 12-14). The list is sorted based on the GScore in descending order so that roots with higher GScores are likely to be visited early (Line 15). Then the procedure recursively calls itself to handle every $r^{\prime}$ in $M$. After all the roots in $M$ are examined if $r$ is still not pruned, we can be sure that $G(r)$ is not dominated by any other G-Graphs and thus can safely add it to $Q$ (Line 1718). The procedure $G_{-}$Prune exists the recursion when the given root $r$ is pruned or added to $Q$.

Figure 5(b) shows how the roots are examined in the case described above. When $G_{-}$Prune is called to handle $r$, it finds that $G\left(r^{\prime}\right)$ has a higher GScore. Then G_Prune is called recursively to handle $r^{\prime}$ (Step 1) and then discovered $r^{\prime \prime}$ (Step 2). After $G\left(r^{\prime \prime}\right)$ has been pushed into $Q$, the procedure prunes all the roots dominated by $r^{\prime \prime}$ (including $r^{\prime}$ ) then returns to the previous call (Step 3). The procedure called to handle $r^{\prime}$ found that $r^{\prime}$ had been pruned when $r^{\prime \prime}$ was handled. Then it returns to the previous call (Step 4). 


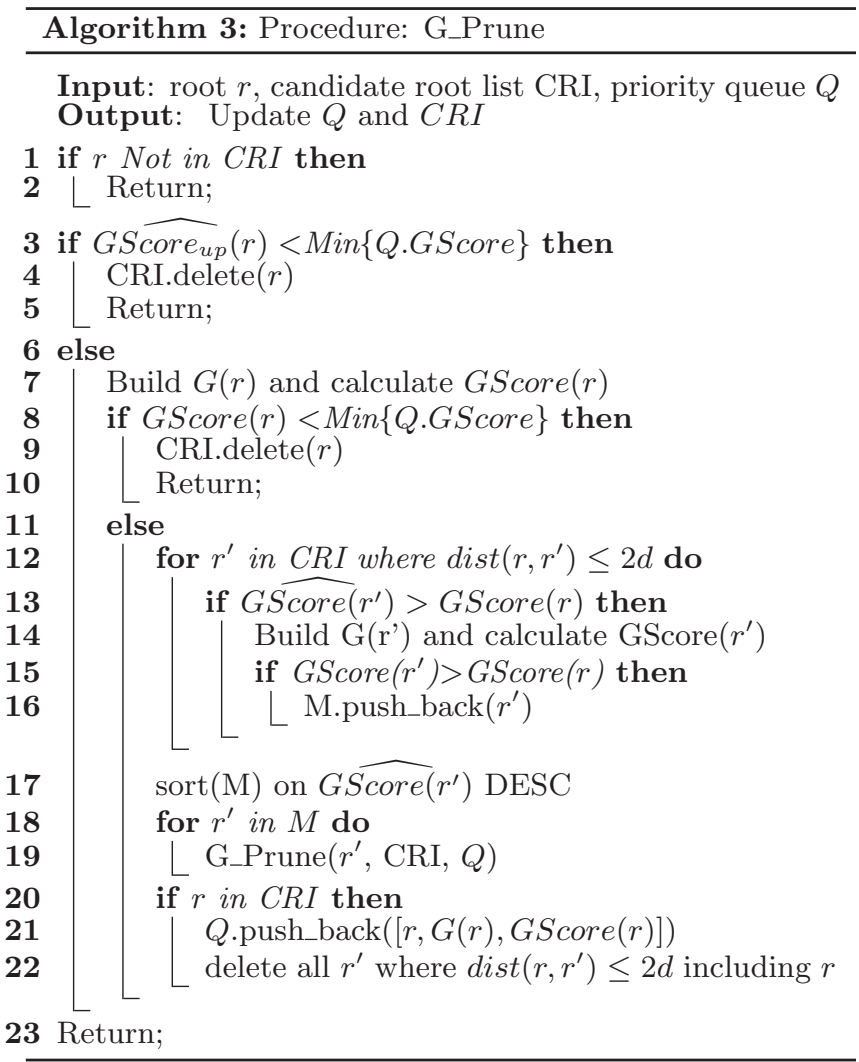

Finally, the procedure called to handle $r$ found that $r$ is not pruned (since $r^{\prime}$ got pruned earlier), $G(r)$ is added to $Q$.

The full SmartEdge algorithm is presented in Algorithm 4. The CRI index and the zone-index are created and updates when the flows are examined to identify the significant ones (Line 2). Then the CRI is sorted based on total incoming significant flows in descending order (Line 3). Finally, each candidate root in CRI will be handled by the G_Prune procedure to generate the final results. There is no postprocessing step needed for SmartEdge.

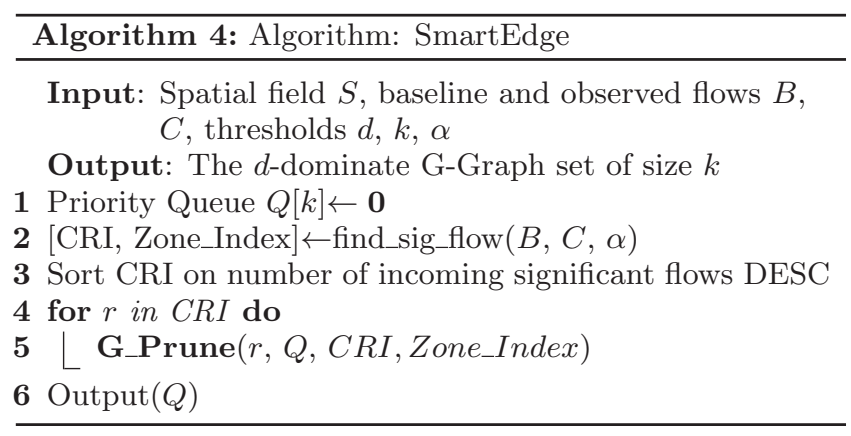

\subsection{Theoretical Analysis}

Both the Brute-Force algorithm and the SmartEdge algorithm need to go through each edge to identify significant flows, which takes $O(|S|)$ time. To build a G-Graph, the brute-force algorithm enumerates all the possible paths from each significant flow to the current root. There are up to $\left(\begin{array}{c}d \\ d / 2\end{array}\right)$ routes and the total cost for this step is up to
Table 1: Time Cost Comparison for the Algorithms

\begin{tabular}{|l|l|l|l|}
\hline Steps & Brute-Force & $\begin{array}{l}\text { SmartEdge } \\
\text { Best }\end{array}$ & $\begin{array}{l}\text { SmartEdge } \\
\text { Worst }\end{array}$ \\
\hline $\begin{array}{l}\text { G-Graphs } \\
\text { Built }\end{array}$ & $|S|$ & $k$ & $X$ \\
\hline $\begin{array}{l}\text { G-Graph } \\
\text { Build cost }\end{array}$ & $O\left(N d \cdot(d)^{d / 2}\right)$ & $O\left(N d^{2}\right)$ & $O\left(N d^{2}\right)$ \\
\hline $\begin{array}{l}\text { Upperbounds } \\
\text { Calculated }\end{array}$ & N/A & $X-k$ & $X$ \\
\hline $\begin{array}{l}\text { Upperbound } \\
\text { calculation } \\
\text { cost }\end{array}$ & N/A & $O(N+d)$ & $O(N+d)$ \\
\hline $\begin{array}{l}\text { Post- } \\
\text { processing }\end{array}$ & $\begin{array}{l}O(|S| \log |S|+ \\
k \cdot|S|)\end{array}$ & None & None \\
\hline Total & $\begin{array}{l}O(|S|(\log |S|+ \\
\left.k+N(d)^{d / 2}\right)\end{array}$ & $\begin{array}{l}O\left(|S|+k N d^{2}\right. \\
+X(N+d))\end{array}$ & $\begin{array}{l}O(|S| \\
\left.X N d^{2}\right)\end{array}$ \\
\hline
\end{tabular}

$O\left(N d(d)^{d / 2}\right)$, where $N$ is the number of significant flows near each root. This is done for all the $|S|$ grids. Then it sorts the G-Graphs based on their GScores and goes through the sorted list to find the $k$-dominant set, leading to a postprocessing overhead of $O(k \cdot|S|+|S| \log |S|)$. The overall time complexity is $O\left(|S|\left(N d \cdot\left(d^{d / 2}\right)\right)+k \cdot|S|+|S| \log |S|\right)$

The SmartEdge Algorithm only examines the possible roots of G-Graphs. We assume at least $k$ G-Graphs can be built. Building the candidate root index will take $O(|S|)$ time. Using the dynamic programming design decision, a G-Graph can be built with $O(N+d)$ time ( $N$ for checking the zoneindex to find $\widehat{L L R\left(e_{i n s}\right)}, d$ for calculating $\left.N_{e}\right)$. In the best case, the first $k$ G-Graphs found are actually the final results. All the other G-Graphs can be pruned without being generated. The total number of G-Graphs built is thus $k$. The total number of upper-bound calculated is $X-k$ where $X$ is the total number of possible roots, $X<|S|$. In the worst case, the GScore upper-bound of every G-Graph need to be calculated but none of them could be pruned based on the upper-bounds. As a result, all the G-Graphs will have to be built. The total upper-bound calculation and G-Graph generation are both $X$. However, the SmartEdge algorithm does not have any post-processing overhead cost. Table 1 shows the comparison between the two algorithms in different scenarios.

\section{CASE STUDY}

\subsection{Data and Settings}

This section presents a case study to validate the quality of patterns discovered by our proposed method. The dataset we use contains detailed trajectories of over 10,000 taxis in Shenzhen, China, during the 31 days of August, 2013. We partition the city into 500 meter by 500 meter grids. The whole city is thus partitioned into 128 by 64 grids. Choosing smaller grid size will make it hard to track the taxis moving between adjacent grids due to the low GPS sampling rate ( 40 seconds). Then we count the total number of occupied taxis crossing each grid boundary during every 10-minute time windows and generated the flow function used for our problem. If the same taxi have two consecutive GPS points in two neighboring grids, then we add 1 to the boundary flow count. The baseline flows $(B)$ are generated by averaging the monthly average traffic flow crossing the same boundary during the same time of day. Since weekends and weekdays usually have different traffic patterns, we created separated baselines for Friday/Saturdays, Sundays, and other weekdays.

We run the SmartEdge algorithm on the whole month's 


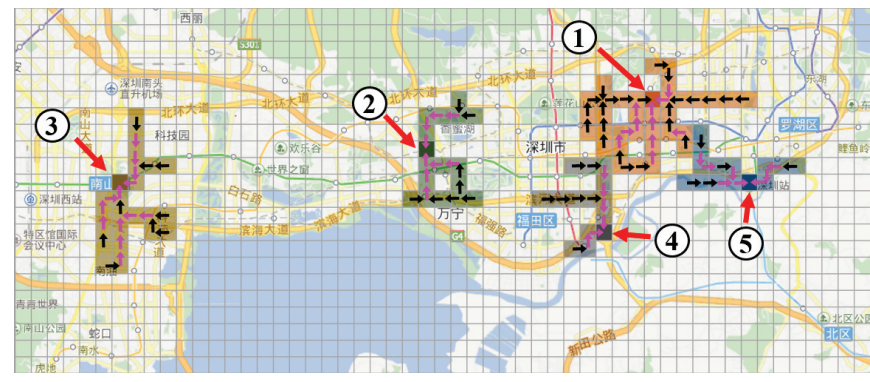

Figure 7: Top-5 G-Graphs, 19:30-19:40, 8/16/2013

data and identify the top 5 most likely gathering events for every tine 10-minute time interval. The most interesting ones are verify by news and reported in this paper. The maximum distance threshold $d$ is set to 5 grids $(2.5 \mathrm{~km})$, and the statistical significance threshold $\alpha=0.001$.

\subsection{Gathering Event On Real Trajectory Data}

On August 16 (Friday) at 8PM, there was a big charity pop concert held in the Shenzhen City Stadium. The stadium has a capacity of 35,000 and was almost filled up despite of the heavy rain that night [14]. Several famous Chinese pop stars performed in the concert. Figure 6 shows the result of our algorithm on the same day in 7 consecutive 10-minute time intervals between 19:20 and 20:30. The most likely gathering destination (orange grid) is very close to the stadium (blue oval). The black arrows and pink arrows represent the identified significant flows and insignificant flows, respectively. The G-Graph near the stadium remains the most likely gathering event with highest GScore in all the 7 time intervals. The footprints clearly showed that big waves of audiences started to arrive half an hour before the concert started. Most traffic gathered towards the destination along the east-west road (Sungang Road) before 8PM. More traffic started to emerge from the South and North after the concert began. The GScore increase from 329.18 at 19:30 to 633.92 at 20:00 when the concert began, then dropped to 506.46 at 20:10 since a big wave of audiences had arrived before the concert began. Then it raised to 717.44 again, suggesting that another big wave of audiences are arriving at the stadium. The root moves to the east after 20:30 and the G-Graph vanished after 20:40.

We also implemented the method from a related work [3] and run it on the data for the same event to compare results. Since their black hole detection algorithm is designed for spatial networks, we treat each grid in our data as a road segment. The actual flow threshold is set to $50 \%$ of the actual flow of the grid we identified as the root between 19:50 and 20:00. Figure 6(h) show that their method discovered a black hole area with high net incoming traffic. However there is no directional information and no gathering destination can be identified.

Figure 7 shows a big picture with the top- 5 most likely gathering destinations and their G-Graphs on August 16, 2013 between 7:30PM and 7:40PM. Grids of each G-Graph are highlighted using different colors. Besides No.1 (the stadium), No.5 is close to Shenzhen Railway Station, No.4 is a port from Shenzhen to Hong Kong. No.2 and No.3 are close to major highway ramps and subway stations where congestion are likely to occur.

\section{EXPERIMENTAL EVALUATIONS}

This section presents our experimental evaluations on the efficiency of the proposed algorithms.

Experiment Settings: The dataset used is the same as the one in the Case Study section. We compare the time cost of the Brute-Force algorithm with the SmartEdge algorithm. For the SmartEdge algorithm we also test its running time with the following combinations of the design decisions: (1) only candidate root filtering (CRF), (2) CRF and G-Graph building with Dynamic Programming $(\mathrm{CRF}+\mathrm{DP})$, and (3) CRF, DP and the G-Graph Pruning $(\mathrm{CRF}+\mathrm{DP}+\mathrm{GPR})$. This way we examine how each design decision impacts the running time. Note that, the third setting is in fact the full SmartEdge algorithm. We don't compare our running time with that of the related work (e.g., [3]) due to different outputs. The algorithms are fed with the whole month's data. We filter time intervals with less than 5 G-Graphs and use the rest 1400 time intervals. In each case we run the same experiment 4 times and report the average CPU time. The experiment is run in Linux on a single Intel Xeon E5-2650 2.00GHz CPU of a cluster with 32 identical CPUs, 20MB Cache for each CPU, and 32GB shared memory.

Through the experiments we hope to answer the following questions: (1) How will the computation time change when varying the number of grids $|\mathrm{S}|$ ? (2) How will the computation time change when varying the statistical significance level $\alpha$ ? (3)How will the computation time change when varying $k$ ? The default parameters are: $|S|=64 \times 128$, $d=5, k=5, \alpha=0.01 \%=0.0001$.

\subsection{Effect of Varying Number of Grids $|S|$}

First we test the impact of varying the number of grids in the study area. We take a sub-area with $2: 1$ side ratio from the center of $S$ and grow it to the full $S$. The number of grids on the short side $L=\sqrt{|S| / 2}$ is changed from 32 to 64 with a step of 8 . The total grids changes from $32 \times 64$ to $64 \times 128$. Other parameters are fixed at default values. Figure 8 (a) shows the results. Brute-Force algorithm runtime increases at linear speed while others increase at a sub-linear speed. This is because SmartEdge filtered impossible roots and G-Graphs but Brute-Force algorithm still examines all the locations in $|S|$. The full SmartEdge can achieve as much as $50 \%$ time savings over the Brute-Force algorithm.

\subsection{Effect of Varying Distance Threshold $d$}

We test the impact of varying $d$ from $1(500 \mathrm{~m})$ to $9(4.5 \mathrm{~km})$. Other parameters are fixed at default values. Increasing $d$ will increase the time to generate G-Graphs for each root. Figure 8(b) shows that both Brute-Force and CRF has an exponential increase speed since they enumerate all the possible paths when generating G-Graphs. The SmartEdge with DP G-Graph Building reduced the cost to super-linear on average case. SmartEdge can achieve on average $49 \%$ time savings over the Brute-Force algorithm. The time savings from GPR is $10 \%$ compared to the version without it.

\subsection{Effect of Varying Result Size $k$}

Next we test the impact of varying the result set size $k$ from 1 (only find the most likely G-Graph) to 10 . Other parameters are fixed at default values. Increasing $k$ will only impact the last algorithm $(\mathrm{CPF}+\mathrm{DP}+\mathrm{GPR})$ since the other three don't use the top-k list to do any pruning. Figure 8 (c) 


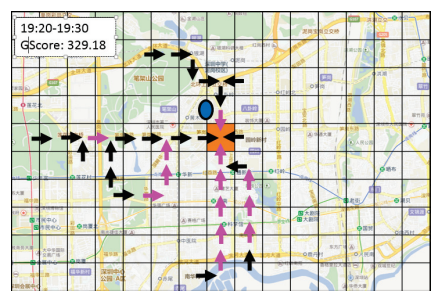

(a) G-Graph at 19:30

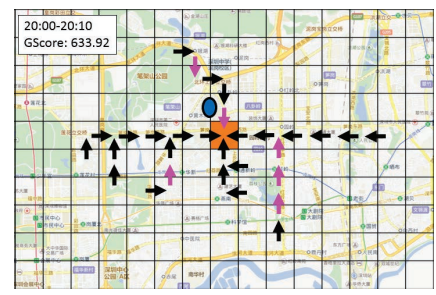

(e) G-Graph at 20:10

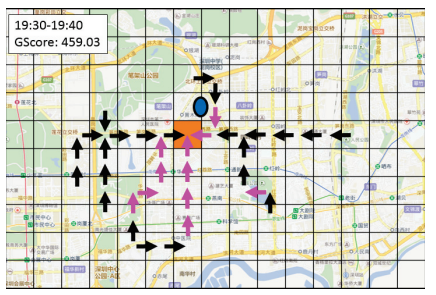

(b) G-Graph at 19:40

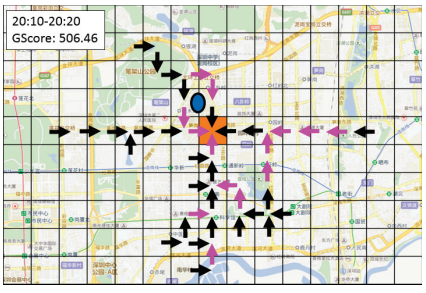

(f) G-Graph at 20:20

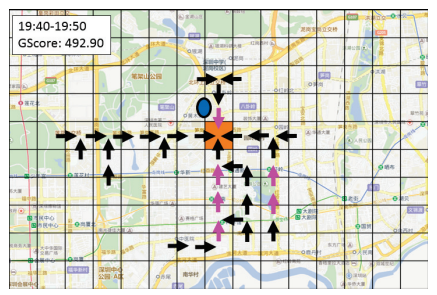

(c) G-Graph at 19:50

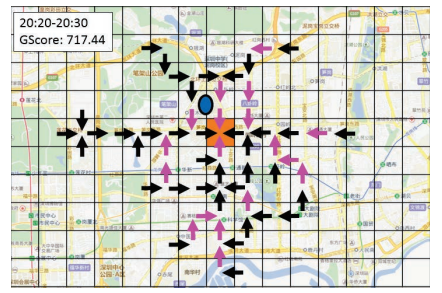

(g) G-Graph at 20:30

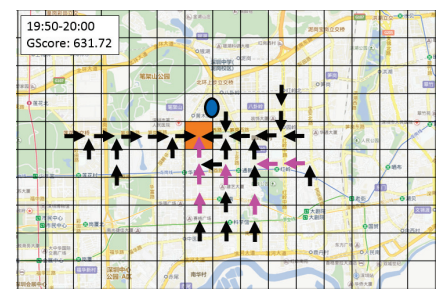

(d) G-Graph at 20:00

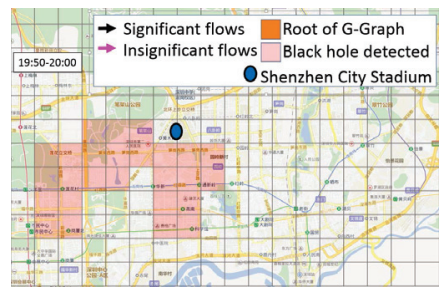

(h) Output of Related Work [3]

Figure 6: Evolving of the Gathering Event near the Stadium

shows that SmartEdge with G-Graph Pruning (green line) has the best performance with smaller $k$. The savings is between $44 \%$ and $50 \%$ over the Brute-Force algorithm and $15 \%$ over the version without GPR. The gap between $\mathrm{CRF}+\mathrm{DP}+$ GPR (green) and CRF+DP (blue) becomes smaller when $k$ increase as the minimum GScore in the top-k list is smaller thus reducing SmartEdge's pruning power.

\subsection{Effect of Varying P-Value Threshold $\alpha$}

Finally we test the impact of varying the p-value threshold $\alpha$ for significant flows from $0.0001(0.01 \%)$ to $0.001(0.1 \%)$ with a step 0.0001 . Increasing $\alpha$ will increase the total number of significant flows $N$ in the complexity. Other parameters are fixed at default values. Figure $8(\mathrm{~d})$ shows that the running time for all the four algorithms increase at a linear speed. However, $\mathrm{CRF}+\mathrm{DP}$ and $\mathrm{CRF}+\mathrm{DP}+\mathrm{GPR}$ grow at a much slower speed. SmartEdge can achieve as much as $52 \%$ time savings over the Brute-Force algorithm and the GPR design decision provided $11 \%$ further improvement over the version without it.

\section{RELATED WORK}

Related work on event detection has focused on representing the footprint of events using regular shapes such as rectangles, circles, or undirected graphs. Based on how events are defined, these work can be further classified into two groups.

The first group of related work identify regions where the total count of objects or instances (e.g., disease cases, vehicles) is higher than expected. Martin Kulldroff's spatial scan statistic [7] is widely used in epidemiology and many other areas. It assumes a distribution (e.g., Poisson) for the number of occurrences of a disease in an area with known population. Then the method examines every possible circular region and calculates the maximum likelihood ratio between the an alternative hypothesis (the risk inside is higher than outside) and the null hypothesis (the risk inside the region is the same as the risk outside). Monte Carlo simulations are used to test the statistical significance of the region with the highest likelihood ratio score. Kulldroff extended his statistic to spatio-temporal setting[4, 5, $6]$, where cylinder-shaped clusters instead of circular clus- ters are used to account for the time span of the outbreak events. Neil et al [10] modified Kulldroff's framework and proposed an Expectation-based Poisson (EBP) model. Instead of comparing the risk inside a region against outside, EBP compares the observed count of a region with its own historical average. Neil et al. also proposed a Bayesian version of the spatial scan statistics $[11,13]$. Additionally, some work focused on speeding up Kulldroff's algorithm. Neill et al. [12] proposed an algorithm that divided rectangular areas into overlapping sub-rectangles and calculated an upper bound of the score in each sub-region for quick pruning. Agarwal et al. [1] proposed a heuristic to approximate the discrepancy function assuming it is convex. Wu et al. [17] proposed a likelihood ratio test framework to find the most likely cluster in a grid with much lower computational cost than exhaustive search.

The second group of related work focused on identifying regions where the incoming traffic is higher than outgoing traffic, or vice versa. Such regions are also called Black-holes and Volcanoes. Li et al. $[9,8]$ proposed a framework to model blackholes and an algorithm to discover top-k blackhole subgraphs and applied the method to financial data in a purely spatial setting. Hong et al. [3] applied the same idea to an urban setting. Their work identifies sub-graphs with net traffic (incoming minus outgoing) higher than predefined thresholds. There are a few issues that were not addressed by these work: (1) they do not compare the observed traffic with any baseline thus may find trivial events such as morning rush hour congestion, (2) using the same net traffic threshold for the entire region and time period is inappropriate since traffic density are heterogeneous over space and time, and (3) the results of these methods don't reflect the direction and path of the gathering traffic.

All of the above related work find undirected footprints of events. While some of them can reflect the impacted area of an event (e.g., black holes), it is hard to tell how traffic flows and gathers inside the impacted region. Our work, by contrast, identify the most likely gathering destination and the path along which moving objects gather towards the destination. Since the outputs of our work and related work are different, we didn't compare the running time of 


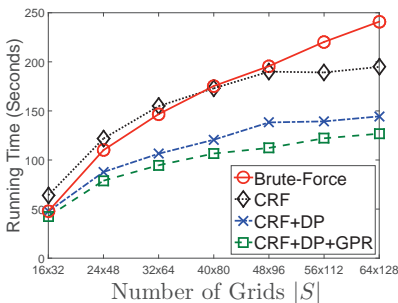

(a) Runtime with varying $|S|$

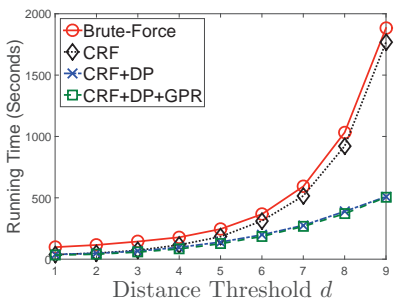

(b) Runtime with varying $d$

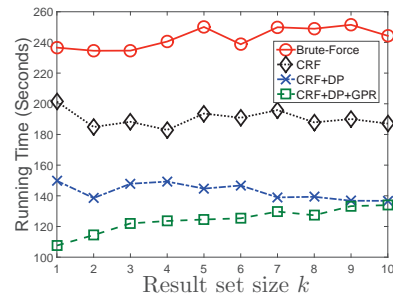

(c) Runtime with varying $k$

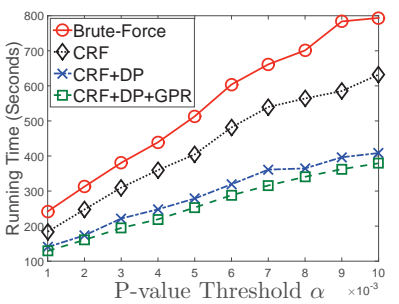

(d) Runtime with varying $\alpha$

Figure 8: Runtime Evaluation Results

our algorithm with that of the related techniques.

Other work such as detecting moving clusters, flocks, or dynamic groups of objects $[18,19,15,2]$ focused on mining the relationship among moving objects instead of footprints of events thus not directly related to the topic of this paper.

\section{CONCLUSION AND FUTURE WORK}

This paper investigated the problem of early detection of gathering events (EDGE). The EDGE problem is important to a broad range of applications in public safety and transportation management, yet it is computationally challenging. Related work did not consider the direction of gathering traffic flow thus can't precisely show the destination of the gathering. This paper formulated the footprint of a gathering event as a Gathering Directed Acyclic Graph (GGraph). An efficient algorithm SmartEdge was proposed to efficiently identify top- $k$ non-overlapping G-Graphs for each time interval. Case studies show that the proposed model and algorithm have the ability to capture important gathering events in real data. Experimental evaluations show that SmartEdge saves $50 \%$ time over the baseline algorithm.

In the future, we plan to (1) do more case studies to find gathering events, (2) use simulation to quantitatively evaluate the accuracy of the destinations detected, and (3) study how to set the the parameters properly.

\section{ACKNOWLEDGMENTS}

This work is partially supported by the National Science Foundation under Grant Numbers 1566386, CNS-1318563, CNS-1524698, CNS-1421407, and IIP-1632051, the National Natural Science Foundation of China under Grant Numbers 61472184 and 61321491, the Jiangsu Innovation and Entrepreneurship (Shuangchuang) Program, the China National Basic Research Program (973 Program) under Grant 2015CB352400, the Research Program of Shenzhen under Grants JSGG20150512145714248, KQCX2015040111035011 and CYZZ20150403111012661, and the Obermann Center for Advanced Studies Interdisciplinary Research Grant at the University of Iowa.

\section{REFERENCES}

[1] D. Agarwal, A. McGregor, J. M. Phillips, S. Venkatasubramanian, and Z. Zhu. Spatial scan statistics: approximations and performance study. In Proceedings of the 12th ACM SIGKDD international conference on Knowledge discovery and data mining, pages 24-33. ACM, 2006.

[2] J. Gudmundsson and M. van Kreveld. Computing longest duration flocks in trajectory data. In Proceedings of the 14 th annual ACM international symposium on Advances in geographic information systems, pages 35-42. ACM, 2006.

[3] L. Hong, Y. Zheng, D. Yung, J. Shang, and L. Zou. Detecting urban black holes based on human mobility data.
In Proceedings of the 23rd SIGSPATIAL International Conference on Advances in Geographic Information Systems, page 35. ACM, 2015.

[4] M. Kulldorff. Prospective time periodic geographical disease surveillance using a scan statistic. Journal of the Royal Statistical Society: Series A (Statistics in Society), 164(1):61-72, 2001.

[5] M. Kulldorff, W. F. Athas, E. J. Feurer, B. A. Miller, and C. R. Key. Evaluating cluster alarms: a space-time scan statistic and brain cancer in los alamos, new mexico. American journal of public health, 88(9):1377-1380, 1998.

[6] M. Kulldorff, R. Heffernan, J. Hartman, R. Assunçao, and F. Mostashari. A space-time permutation scan statistic for disease outbreak detection. PLoS medicine, 2(3):216, 2005.

[7] M. Kulldroff. A spatial scan statistic. Communications in Statistics - Theory and Methods, 26(6):1481-1496, 1997.

[8] Z. Li, H. Xiong, and Y. Liu. Mining blackhole and volcano patterns in directed graphs: a general approach. Data Mining and Knowledge Discovery, 25(3):577-602, 2012.

[9] Z. Li, H. Xiong, Y. Liu, and A. Zhou. Detecting blackhole and volcano patterns in directed networks. In 2010 IEEE International Conference on Data Mining, pages 294-303. IEEE, 2010.

[10] D. B. Neill. Expectation-based scan statistics for monitoring spatial time series data. International Journal of Forecasting, 25(3):498-517, 2009.

[11] D. B. Neill and G. F. Cooper. A multivariate bayesian scan statistic for early event detection and characterization. Machine learning, 79(3):261-282, 2010.

[12] D. B. Neill and A. W. Moore. Rapid detection of significant spatial clusters. In Proc. Tenth ACM SIGKDD international conference on Knowledge discovery and data mining, pages 256-265. ACM, 2004.

[13] D. B. Neill, A. W. Moore, and G. F. Cooper. A bayesian spatial scan statistic. Advances in neural information processing systems, 18:1003, 2006.

[14] Sina Entertainment News. http://ent.sina.com.cn/y/2013-08-20/15363991794.shtml.

[15] M. R. Vieira, P. Bakalov, and V. J. Tsotras. On-line discovery of flock patterns in spatio-temporal data. In Proceedings of the 17th ACM SIGSPATIAL international conference on advances in geographic information systems, pages 286-295. ACM, 2009.

[16] Wikipedia. 2014 shanghai stampede - wikipedia, the free encyclopedia, 2016. [Online; accessed 28-June-2016].

[17] M. Wu, X. Song, C. Jermaine, S. Ranka, and J. Gums. A lrt framework for fast spatial anomaly detection. In Proceedings of the 15th ACM SIGKDD international conference on Knowledge discovery and data mining, pages 887-896. ACM, 2009.

[18] K. Zheng, Y. Zheng, N. J. Yuan, and S. Shang. On discovery of gathering patterns from trajectories. In Data Engineering (ICDE), 2013 IEEE 29th International Conference on, pages 242-253. IEEE, 2013.

[19] K. Zheng, Y. Zheng, N. J. Yuan, S. Shang, and X. Zhou. Online discovery of gathering patterns over trajectories. IEEE Transactions on Knowledge and Data Engineering, 26(8):1974-1988, 2014. 